\title{
Immunopositivity for Histone MacroH2A1 Isoforms Marks Steatosis-Associated Hepatocellular Carcinoma
}

\author{
Francesca Rappa ${ }^{1,29}$, Azzura Greco ${ }^{39}$, Christine Podrini ${ }^{3}$, Francesco Cappello ${ }^{1,2,4}$, Michelangelo Foti ${ }^{5}$, \\ Lucie Bourgoin ${ }^{5}$, Marion Peyrou ${ }^{5}$, Arianna Marino ${ }^{6}$, Nunzia Scibetta ${ }^{7}$, Roger Williams ${ }^{3}$, \\ Gianluigi Mazzoccoli ${ }^{8}$, Massimo Federici ${ }^{6}$, Valerio Pazienza9 ${ }^{9 \uparrow}$, Manlio Vinciguerra ${ }^{2,3^{* ศ / x}}$
}

1 Department of Experimental Biomedicine and Clinical Neurosciences, Section of Human Anatomy, University of Palermo, Palermo, Italy, 2 Euro-Mediterranean Institute of Science and Technology, Palermo, Italy, $\mathbf{3}$ Institute of Hepatology, Foundation for Liver Research, London, United Kingdom, $\mathbf{4}$ Istituto “Paolo Sotgiu, Libera Università degli Studi di Scienze Umane e Tecnologiche, Lugano, Switzerland, 5 Department of Cell Physiology and Metabolism, University of Geneva, Geneva, Switzerland, 6 Department of Systems Medicine, University of Rome Tor Vergata, Rome, Italy, 7 Pathologic Anatomy Unit, Civic Hospital, Palermo, Italy, 8 Department of Medical Sciences, Division of Internal Medicine and Chronobiology Unit, IRCCS "Casa Sollievo della Sofferenza” Hospital, San Giovanni Rotondo, Italy, 9 Division and Laboratory of Gastroenterology, IRCCS “Casa Sollievo della Sofferenza" Hospital, San Giovanni Rotondo, Italy

\begin{abstract}
Background: Hepatocellular carcinoma (HCC) is one of the most common cancers worldwide. Prevention and risk reduction are important and the identification of specific biomarkers for early diagnosis of HCC represents an active field of research. Increasing evidence indicates that fat accumulation in the liver, defined as hepatosteatosis, is an independent and strong risk factor for developing an HCC. MacroH2A1, a histone protein generally associated with the repressed regions of chromosomes, is involved in hepatic lipid metabolism and is present in two alternative spliced isoforms, macroH2A 1.1 and macroH2A1.2. These isoforms have been shown to predict lung and colon cancer recurrence but to our knowledge, their role in fatty-liver associated HCC has not been investigated previously.
\end{abstract}

Methods: We examined macroH2A1.1 and macroH2A1.2 protein expression levels in the liver of two murine models of fatassociated HCC, the high fat diet/diethylnistrosamine (DEN) and the phosphatase and tensin homolog (PTEN) liver specific knock-out (KO) mouse, and in human liver samples of subjects with steatosis or HCC, using immunoblotting and immunohistochemistry.

Results: Protein levels for both macroH2A1 isoforms were massively upregulated in $\mathrm{HCC}$, whereas macroH2A1.2 was specifically upregulated in steatosis. In addition, examination of human liver samples showed a significant difference $(\mathrm{p}<0.01)$ in number of positive nuclei in HCC $(100 \%$ of tumor cells positive for either macroH2A1.1 or macroH2A 1.2$)$, when compared to steatosis ( $<2 \%$ of hepatocytes positive for either isoform). The steatotic areas flanking the tumors were highly immunopositive for macroH2A1.1 and macroH2A1.2.

Conclusions: These data obtained in mice and humans suggest that both macroH2A 1 isoforms may play a role in HCC pathogenesis and moreover may be considered as novel diagnostic markers for human HCC.

Citation: Rappa F, Greco A, Podrini C, Cappello F, Foti M, et al. (2013) Immunopositivity for Histone MacroH2A1 Isoforms Marks Steatosis-Associated Hepatocellular Carcinoma. PLoS ONE 8(1): e54458. doi:10.1371/journal.pone.0054458

Editor: Franco Folli, University of Texas Health Science Center at San Antonio, United States of America

Received November 6, 2012; Accepted December 11, 2012; Published January 23, 2013

Copyright: (c) 2013 Rappa et al. This is an open-access article distributed under the terms of the Creative Commons Attribution License, which permits unrestricted use, distribution, and reproduction in any medium, provided the original author and source are credited.

Funding: The authors have no support or funding to report.

Competing Interests: Manlio Vinciguerra is a PLOS ONE Editorial Board member. This does not alter the authors' adherence to all the PLOS ONE policies on sharing data and materials.

*E-mail: manliovinciguerra@iemest.eu

a Current address: Institute for Liver and Digestive Health, Division of Medicine, University College London (UCL), London, United Kingdom

9 These authors contributed equally to this work.

- These authors also contributed equally to this work

\section{Introduction}

The current pandemic in obesity/metabolic syndrome is a risk factor for many types of cancer. The largest increase in cancer risk $(\sim 5$ fold) in obese individuals with high body mass index (BMI $35-$ 40) was seen for primary hepatocellular carcinoma (HCC) [1]. Obesity is accompanied in up to $90 \%$ of cases by non-alcoholic fatty liver disease (NAFLD) [2]. The latter is the consequence of an imbalance between lipid availability through fatty acid uptake and de novo lipogenesis, and lipid secretion and disposal via free fatty acid oxidation, resulting in hepatic accumulation of lipids (steatosis) [3]. In $10 \%$ of the cases NAFLD will progress to a steatohepatitis (NASH), and in $8-26 \%$ to cirrhosis, with an increasingly reported percentage of cases with cirrhosis or NAFLD at an earlier stage developing HCC [4]. Recent studies in rodent models suggested that high fat in the liver might trigger the development of HCG through inflammation, activating specific signalling pathways, growth factors and cytokines $[5,6,7,8,9,10]$. 
Alterations in hepatocyte metabolism and proliferation during steatosis and HCC are triggered by changes in gene transcriptional patterns. The epigenetic mechanisms involved in HCC associated with obesity/metabolic syndrome/steatosis have not been investigated in detail. Nuclear chromatin compaction is regulated at several levels, allowing transcriptional plasticity [11]: one of these is the replacement of canonical histones around which DNA is wrapped (H2A, H2B, H3 and $\mathrm{H} 4)$ with the incorporation of histone variants. The histone variant of histone H2A known as macroH2Al is believed to act as a strong transcriptional modulator that can either repress transcription [12,13], but can also activate a subset of genes in response to as yet undefined growth signals $[14,15]$. MacroH2Al knock out $(\mathrm{KO})$ mice display hepatic steatosis and derangements in glucose and lipid metabolism $[16,17]$ and, interestingly, when wild type mice are fed a methyl-deficient diet, which induce a fatty liver and inflammation, a total increase in the hepatic content of macroH2Al is observed [18].

MacroH2Al is present in 2 isoforms, macroH2A1.1 and macroH2A1.2, which are generated upon RNA alternative exon splicing. The expression of both isoforms has been shown to predict lung cancer recurrence [19] and, in colon cancer, macroH2A1.1 inversely correlates with cancer severity and survival, whereas macroH2A1.2 does not show such correlation [20]. Recently, it has been shown that splicing of macroH2Al isoforms regulates cancer cell growth [21]. In A549 lung cancer cells, HeLa cervical adenocarcinoma cells, IMR90 primary lung fibroblasts, and MG-63 osteosarcoma cells reduced levels of macroH2A1.1 compared to macroH2A1.2 were observed [21]. Reintroduction of macroH2A1.1 suppressed the growth of these cancer cell lines [21]. Other studies, which did not distinguish between the isoforms, demonstrated that $\mathrm{KO}$ of all macroH2Al isoforms induced the progression of the melanoma malignant phenotype both in vitro and in vivo through increased expression of CDK8 oncogene [22].

Regulation by macroH2Al of oncogenes and/or tumor suppressors' expression in hepatocytes could be particularly relevant for fatty liver-associated $\mathrm{HCC}$, since the activities of these genes often link mechanistically hepatic steatosis to the onset of HCG, as we have previously shown for tumor suppressor phosphatase with tensin homology (PTEN) $[23,24,25]$. PTEN is one of the most important tumor suppressors, mutated or deleted in nearly half of human cancers, including HCG patients [26] and changes in its expression have also been shown to regulate hepatic lipid metabolism and insulin sensitivity $[23,24,25,26]$. In this study we explored if an altered expression of macroH2Al isoforms might be associated to fatty liver-associated HCC using two robust mice models for this transition, the dietary high fat/diethynitrosamine (DEN) diet [8] and the genetic liver-specific PTEN knockout $(\mathrm{KO})$ mouse [27]. Furthermore, we examined the expression of macroH2Al isoforms in human liver biopsies from patients where HCC occurred in a background of pure steatosis, in absence of other liver diseases.

\section{Materials and Methods}

\section{Ethics Statement}

Human biopsies: all the procedures followed were in accordance with the ethical standards of the responsible committees (institutional and national) on human experimentation and with the Helsinki Declaration of 1975 (as revised in 2008). Written informed consents were obtained from all patients at the time of biopsy and the study was approved by Ethics Committee of the Civic Hospital, Palermo, Italy.

Mice models: for the PTEN KO model, all the procedures were in accordance with the Swiss guidelines for animal experimentation and with ethically written approval by the Geneva (Switzerland) health head office. For the HF/DEN model, procedures were in accordance with the Italian national authorities and written approval was obtained by the Institutional Animal Care of the University of Rome "Tor Vergata" protocol 28/02/ 2012 n. 17.

\section{Mice Models}

According to an established protocol [8], in the DEN-induced HCC model, DEN $(25 \mathrm{mg} / \mathrm{kg}$ ) was injected intraperitoneally into 14 days old mice. After 4 weeks, mice were separated into two dietary groups and fed either chow or high fat diet until sacrificed at 36 weeks of age. High fat diet (composed of 59\%-fat, 15\%protein, 26\%-carbohydrates based on caloric content) was purchased from Research Diet, New Brunswick, NJ, US.

To obtain PTEN liver specific KO mice, Pten ${ }^{\text {flox/flox }}$ mice $(129$ Ola $\infty \mathrm{C} 57 \mathrm{BL} 6 / \mathrm{J} \mathrm{F} 2)$ were mated to AlbCre transgenic mice (C57BL6/J background) as previously described [27], in which expression of Cre is controlled by the promoter of the hepatocytespecific gene Albumin. Offspring carrying AlbCre and two copies of the floxed Pten allele (AlbCrePten ${ }^{\text {flox/flox }}$ ), and control Pten ${ }^{\text {flox/ }}$ flox mice were retained for experiments. Mice were sacrificed at 16 weeks for studying the steatotic phenotype and at 52 weeks for studying the HCG phenotype. PCR analysis of PTEN genotypes was performed as previously reported [27].

In both high fat diet DEN-induced HCG and PTEN KOinduced HCC models, tumors in each liver lobe were counted. Serum, liver tumor and non-tumor tissue was collected and rapidly frozen for biochemical and histological analyses (see below).

\section{Human Sample Collection}

Formalin-fixed paraffin embedded biopsies were retrospectively collected from files of the Pathologic Anatomy Unit of the Civico Hospital, Palermo, Italy. 10 cases were selected of mild mixed macro- and micro-vesicular steatosis. 10 cases of HCG arising in macro-vesicular steatosis were also selected. The clinical characteristics of the patients studied are summarized in Table 1, in terms of history of either $\mathrm{HBV} / \mathrm{HCV}$ infection, cirrhosis, alcoholism and NAFLD score (see next paragraph). Fibrosis and/or cirrhosis were not observed in the biopsies.

Table 1. Clinical characteristics of the patients studied.

\begin{tabular}{lllllllll}
\hline & & & & & & \\
\hline DISEASE & \# OF CASES & $\begin{array}{l}\text { SEX } \\
\text { (M/F) }\end{array}$ & $\begin{array}{l}\text { AGE RANGE } \\
\text { (mean) }\end{array}$ & HBV & HCV & $\begin{array}{l}\text { NAFLD SCORE* } \\
\text { RANGE }\end{array}$ & CIRRHOSIS & ALCOHOLISM \\
\hline STEATOSIS & 10 & $5 / 5$ & $45-71(59.75)$ & $0 / 10$ & $0 / 10$ & $0-2$ & $0 / 10$ & $0 / 6$ (UNKNOWN IN 4 CASES) \\
HCC & 10 & $5 / 5$ & $40-75(57.90)$ & $0 / 10$ & $0 / 10$ & $0-4$ & $0 / 10$ & $0 / 10$ \\
\hline
\end{tabular}

*Assessed according to Ref. 29.

doi:10.1371/journal.pone.0054458.t001 
A
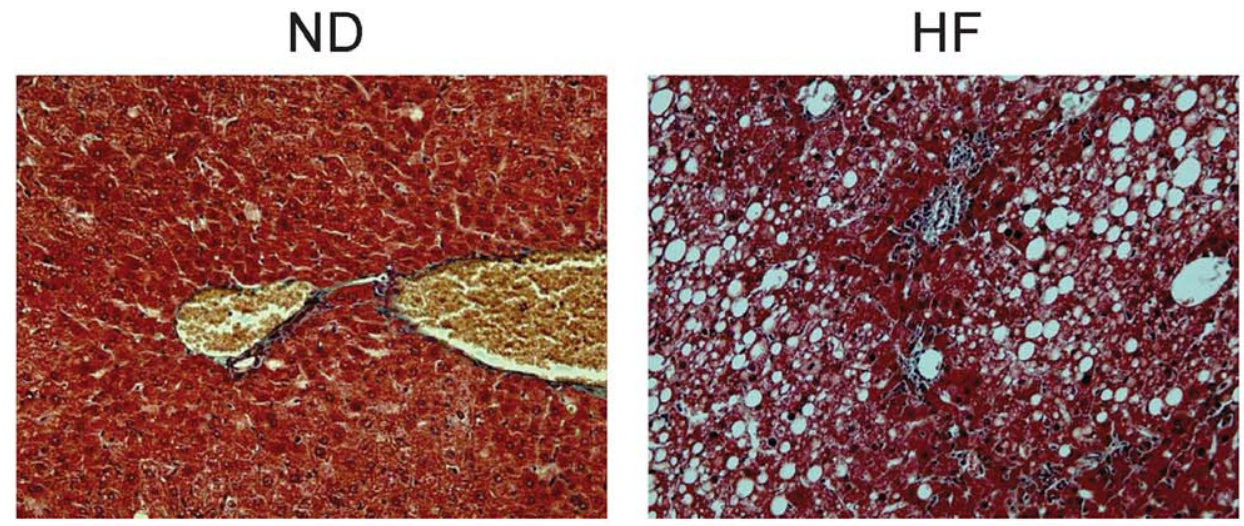

B
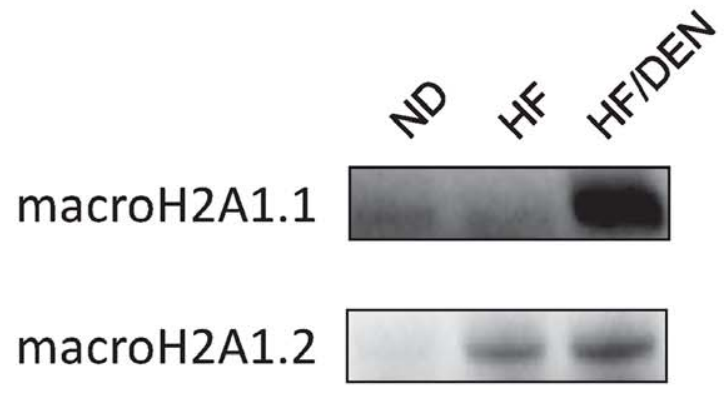

H3

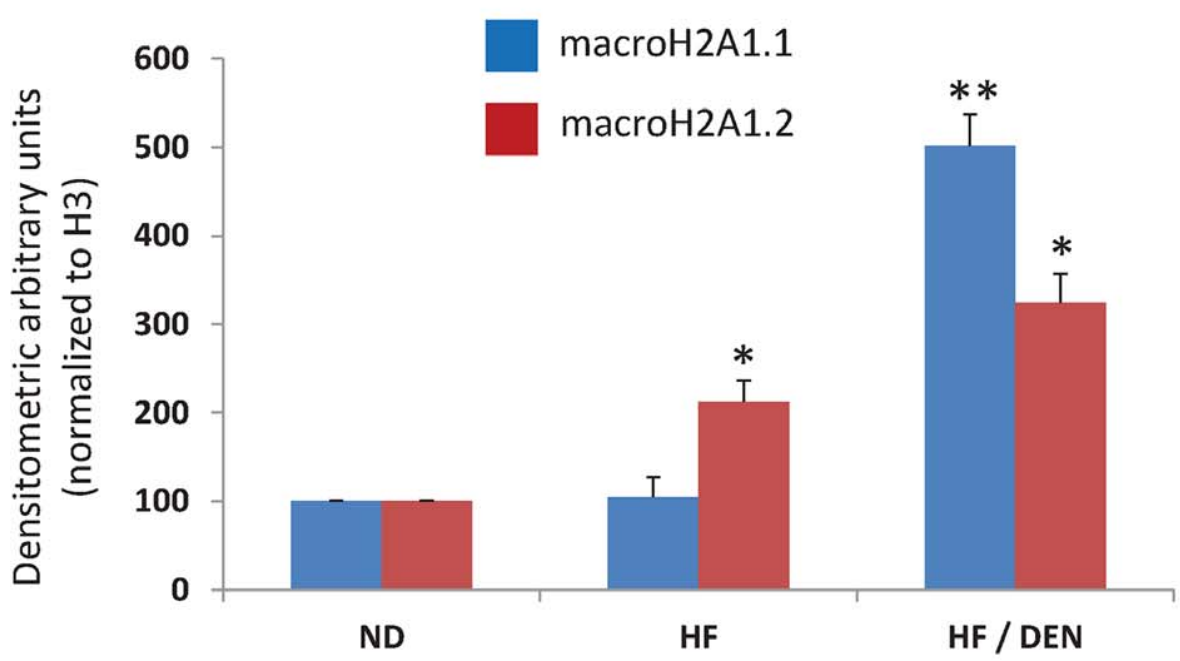

Figure 1. Protein expression of macroH2A1 isoforms in the liver of HF/DEN mouse model of steatosis and HCC. A. Representative pictures of trichrome staining in liver samples from mice fed a normal diet (ND) and mice fed a high fat (HF) diet for 36 weeks. B. Histone extracts from livers of ND, HF and HF/DEN mice were processed for immunoblotting. MacroH2A1.1, macroH2A1.2 and histone H3 (loading control) protein levels were detected with specific antibodies. Representative immunoblots are shown C. Densitometric quantification of macroH2A1.1, macroH2A1.2 protein levels in the livers of $\mathrm{ND}, \mathrm{HF}$ and HF/DEN mice. $\mathrm{N}=5,{ }^{*} \mathrm{P}<0.05,{ }^{*} \mathrm{P}<0.01$ versus ND mice. doi:10.1371/journal.pone.0054458.g001 
A
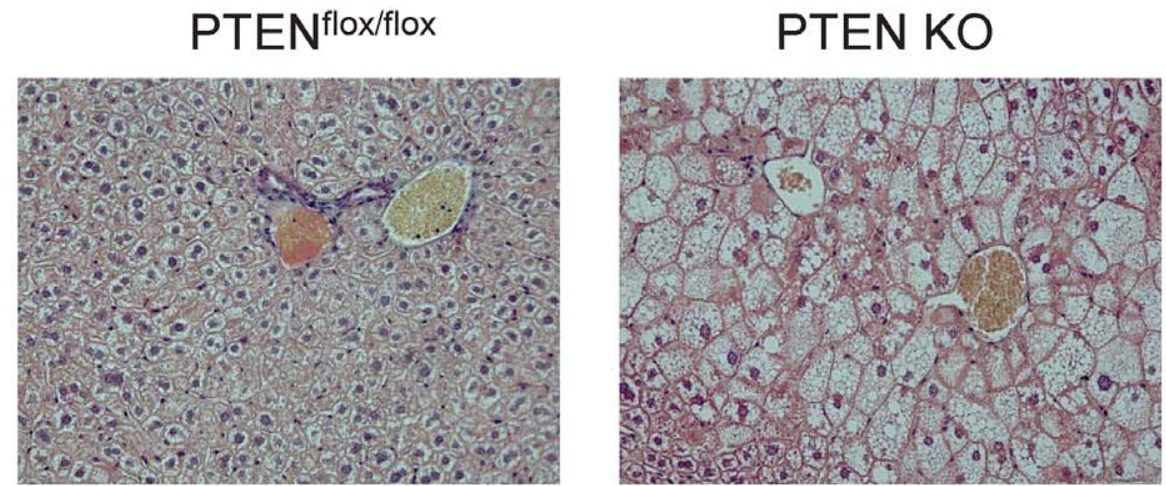

B

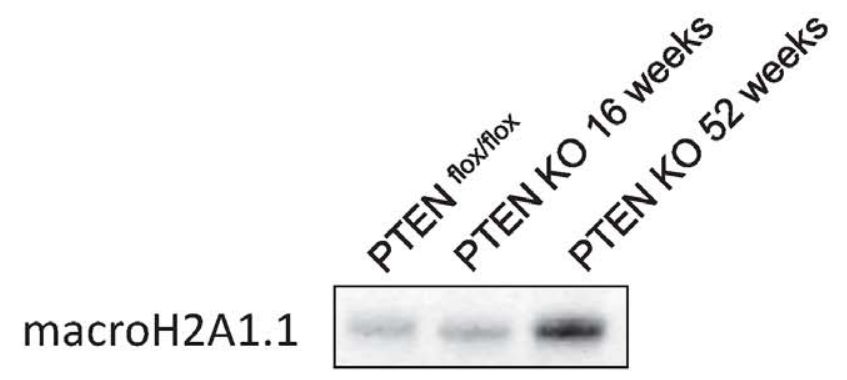

macroH2A1.2

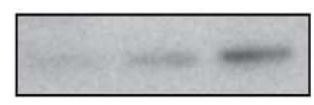

H3

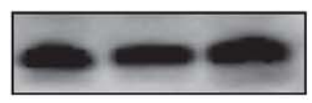

C



Figure 2. Protein expression of macroH2A1 isoforms in the liver of the liver-specific PTEN KO mouse model of steatosis and HCC. A. Representative pictures of trichrome staining in liver samples from PTEN ${ }^{\text {flox/flox }}$ and PTEN KO mice. B. histone extracts from livers of 16 weeks old PTEN $^{\text {flox/flox }}, 16$ weeks old PTEN KO and from liver tumors of 52 weeks old PTEN KO mice were processed for immunoblotting. MacroH2A1.1, macroH2A1.2 and histone H3 (loading control) protein levels were detected with specific antibodies. Representative immunoblots are shown. C. Densitometric quantification of macroH2A1.1, macroH2A1.2 protein levels in the livers of 4 months old PTEN flox/flox $(\mathrm{N}=5), \mathrm{PTEN}$ KO 16 weeks old $(\mathrm{N}=5)$ and in the liver tumors of PTEN KO 52 weeks old mice $(\mathrm{N}=2),{ }^{*} \mathrm{P}<0.05,{ }^{* *} \mathrm{P}<0.01$ versus PTEN ${ }^{\text {flox/flox }}$ mice. doi:10.1371/journal.pone.0054458.g002

\section{Histological Assessment of NAFLD Score}

Sections from both mice and human specimens were processed by haematoxylin and eosin $(\mathrm{H} \& \mathrm{E})$ and Masson trichrome staining for histological evaluation of NAFLD score, as previously described $[28,29]$, in which a semi-quantitative scoring system that grouped histological features into five broad categories of 


\section{Steatosis}
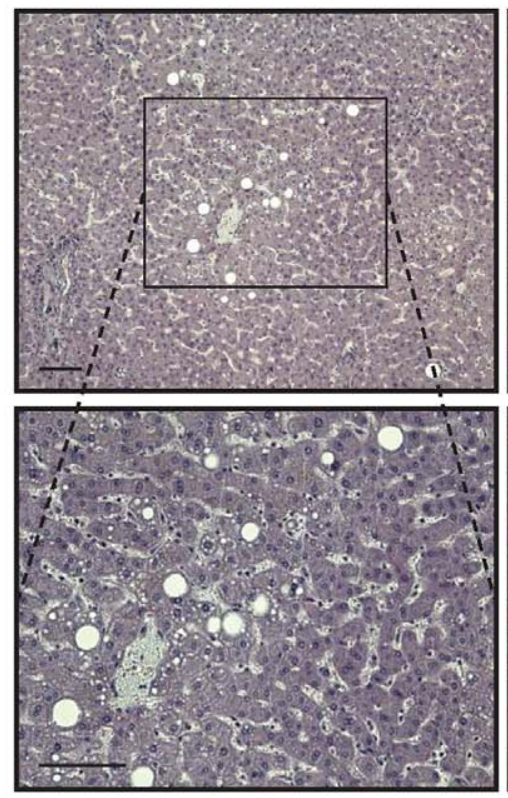

St/HCC
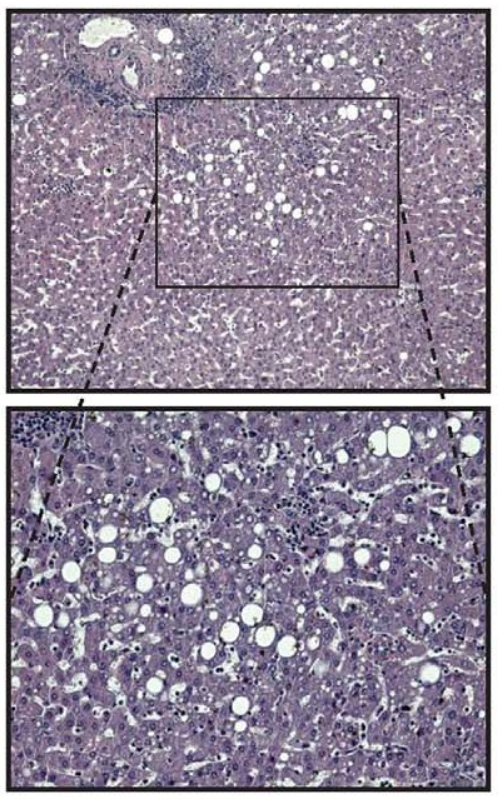

HCC

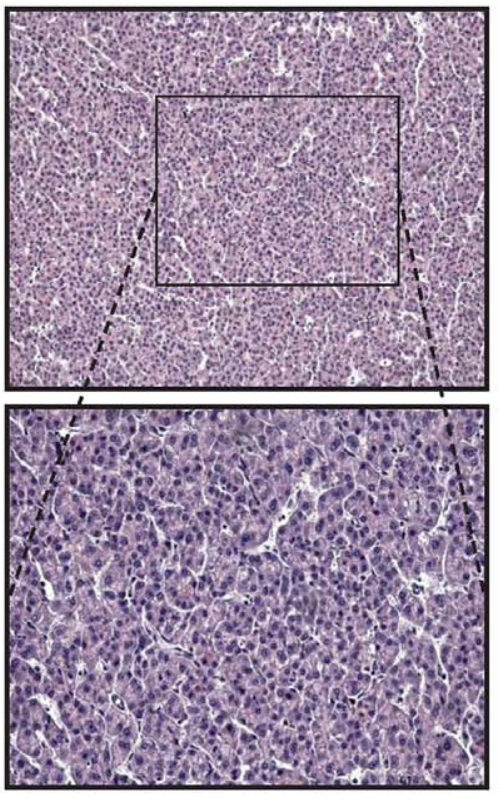

B

\section{Steatosis}

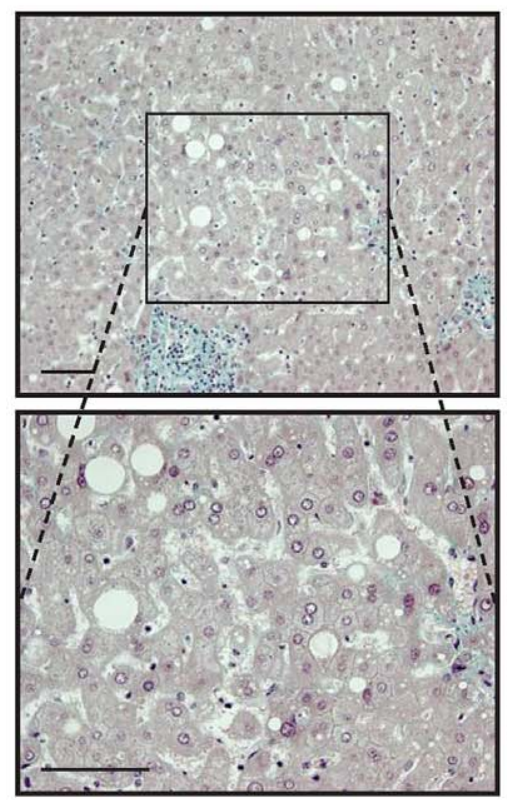

St/HCC
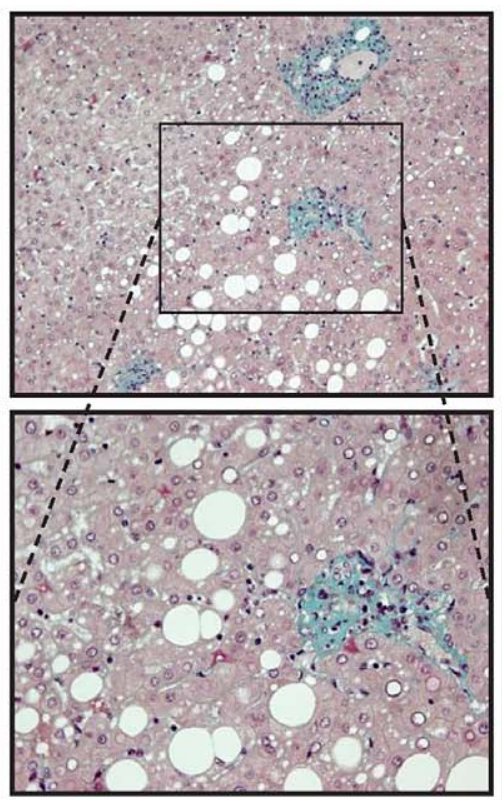

\section{HCC}

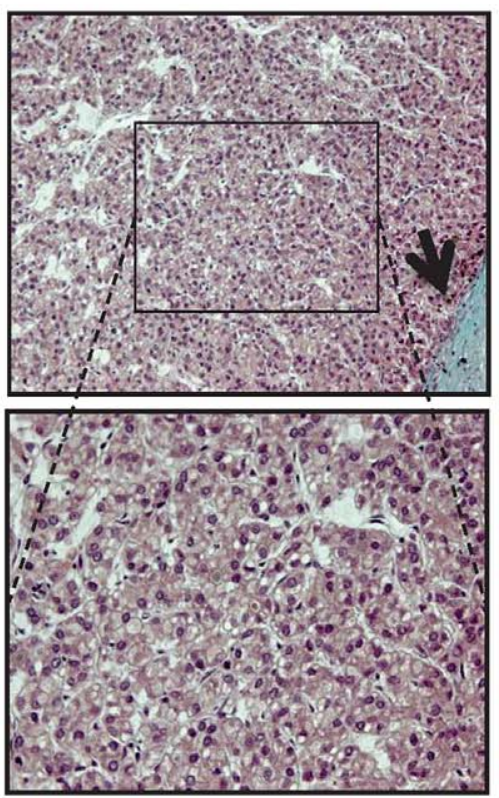

Figure 3. Histological features of liver biopsies of patients with steatosis and HCC. A. Representative pictures of hematoxylin and eosin (H/E) staining. In HCC samples both area with tumor and area of steatosis close to HCC (St/HCC) were examined. Pictures showed the same area observed with a lower (above) and higher (below) magnification. B. Representative pictures of trichrome staining in samples with steatosis and HCC. In the latter both area with tumor and St/HCC were examined. Pictures showed the same area observed with a lower (above) and higher (below) magnification. Trichrome stains showed that collagen (green) was present only in portal space and, in limited amount, in perisinusoidal (Disse) spaces of lobule in both steatosis and St/HCC samples, in which macro and micro vesicular steatosis is also visible. Collagen was also present in correspondence of the capsule that delimits HCC (arrow). Bar: $100 \mu \mathrm{m}$. doi:10.1371/journal.pone.0054458.g003 

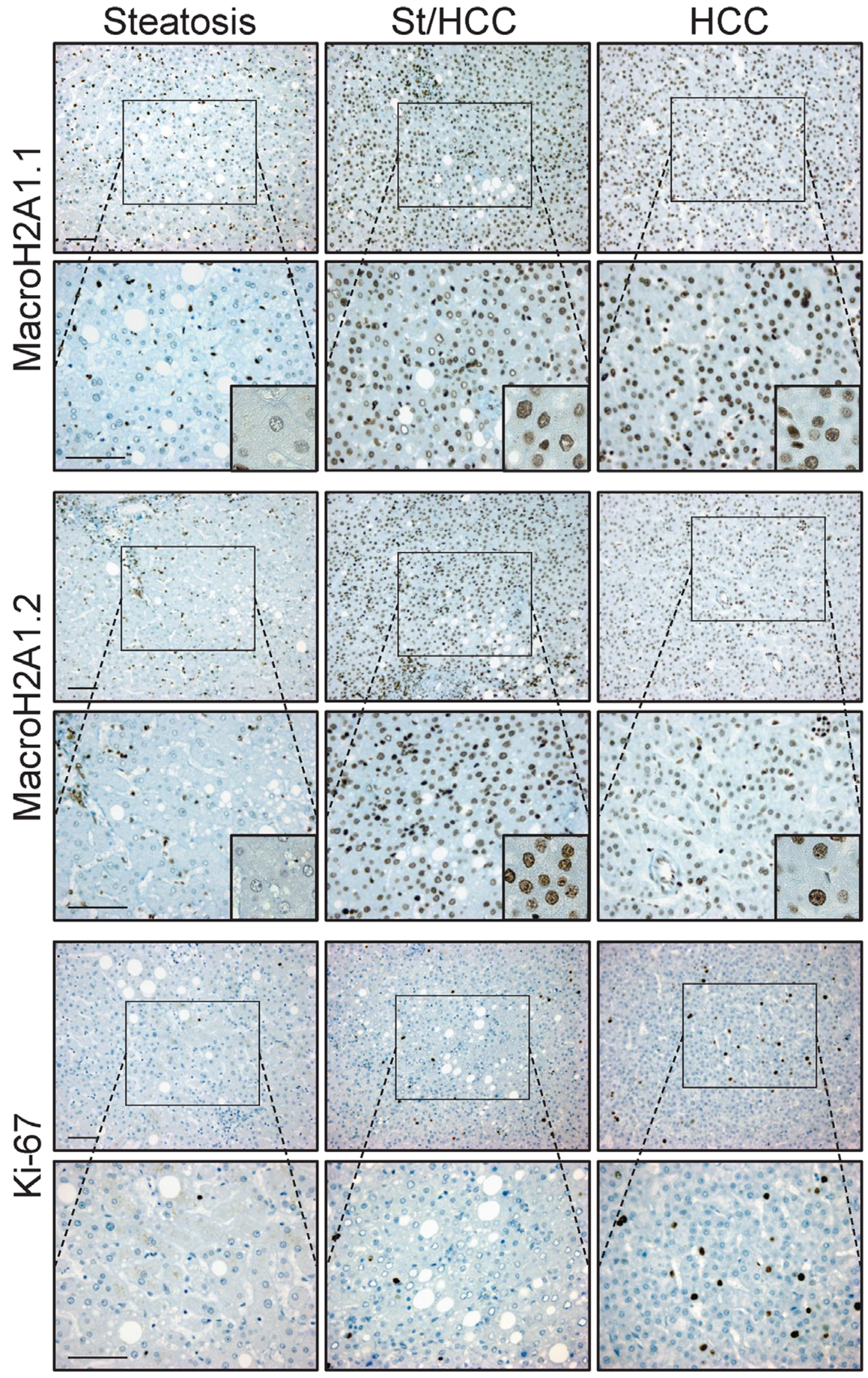
Figure 4. Representative pictures of immunostaining performed for MacroH2A1.1, MacroH2A1.2 and Ki-67 in samples with steatosis and HCC. In the latter both area with tumor and area of steatosis close to HCC (St/HCC) were examined. All nuclei of tumor cells were positive for either macroH2A1.1 or macroH2A1.2. Positivity in hepatocytes of steatosis was significantly lower. Pictures showed the same area observed with a lower (above) and higher (below) magnification. Insets show details of nuclear staining. Bar: $100 \mu \mathrm{m}$.

doi:10.1371/journal.pone.0054458.g004

steatosis, inflammation, hepatocellular injury, fibrosis, and miscellaneous features was performed.

\section{Western Blot Analyses}

Cytoplasmic and nuclear protein extraction from nontumorous liver parenchyma and HCC tissue preparations and immunoblotting analyses were performed as previously described [23,25]. Histone fraction was enriched using an acid extraction protocol. Briefly, The snap-frozen tissues were suspended and homogenized in $200 \mu \mathrm{l}$ of $\mathrm{H}$-lysis solution $(0.2 \mathrm{M}$ sucrose, $3 \mathrm{mM} \mathrm{CaCl} 2,1 \mathrm{mM}$ Tris-HCl pH8.0, 0.5 NP40, protease inhibitor cocktail), incubated on ice for $8 \mathrm{~min}$; centrifuged at $1.300 \times \mathrm{g}, 4^{\circ} \mathrm{C}$, for $5 \mathrm{~min}$ to separate supernatant from nuclei fraction $(\mathrm{P} 1)$. Pl was washed once with $\mathrm{H}$-wash solution $(300 \mathrm{mM} \mathrm{NaCl}, 5 \mathrm{mg} \mathrm{MgCl} 2,5 \mathrm{mM}$ DTT, $0.5 \%$ NP40)and lysed for $30 \mathrm{~min}$ in $100 \mu \mathrm{H}$-extract solution $(0.5 \mathrm{mM} \mathrm{HCl}, 10 \%$ glycerol, protease inhibitor cocktail), followed by centrifugation at $13.000 \times \mathrm{g} 4^{\circ} \mathrm{C}$, for $5 \mathrm{~min}$. Finally, TCA precipitation was performed. Antibodies against histone H3 (Activ Motif) were use to normalize protein levels.

\section{Immunohistochemistry}

Immunostainings were performed by iVIEW DAB Detection Kit for Ventana BenchMark XT automated slide stainer on human biopsies [30]. Primary antibodies for MacroH2A1.1 and MacroH2A1.2 were generated at the European Molecular Biology Laboratory (EMBL) and were a courtesy of Prof. Andreas Ladurner (Ludwig Maximilian University, LMU, Munich, Germany). Positivity for Ki-67 (Vector VP-K451, DBA ITALIA S.R.L., Milan, Italy ) was also examined. All primary antibodies were diluted 1:100. Positive and negative controls were run concurrently. Immunopositive evaluations were performed in blind by three expert pathologists (FR, FG and NS) and percentage of positive nuclei (tumor cells in HCG and hepatocytes in steatosis) was calculated in ten random high power fields at a magnification of 400x.

\section{Statistical Analysis}

Results are expressed as means \pm S.E. Comparisons were made by using Student's t test. Differences were considered as significant when $\mathrm{P}<0.05, \mathrm{P}<0.01$ or $\mathrm{P}<0.001$, as indicated in the Figures and Figure Legends.

\section{Results}

MacroH2A1.1 and macroH2A1.2 Expression in the Liver of High Fat Diet fed/DEN Mice

A potent bona fide dietary mouse model of high fat-induced HCG developed recently was reproduced in this study [8]. Male mice maintained on $\mathrm{HF}$ gained more weight than mice on a normal diet (ND), developed glucose intolerance and their relative liver weight and triglycerides were increased (data not shown) [8]. This was accompanied by increased hepatic steatosis with a mean of NAFLD score of 1 versus 5, respectively (Fig. 1A). Mice fed with $\mathrm{ND}$ and treated with DEN injection at a low dose of $25 \mathrm{mg} / \mathrm{kg}$ did not display steatosis, they were indistinguishable from animal fed a ND (data not shown) and therefore they were not retained for further analyses. At sacrifice, mice injected with DEN and kept on HF exhibited HCG nodules, as well as augmented levels of inflam-
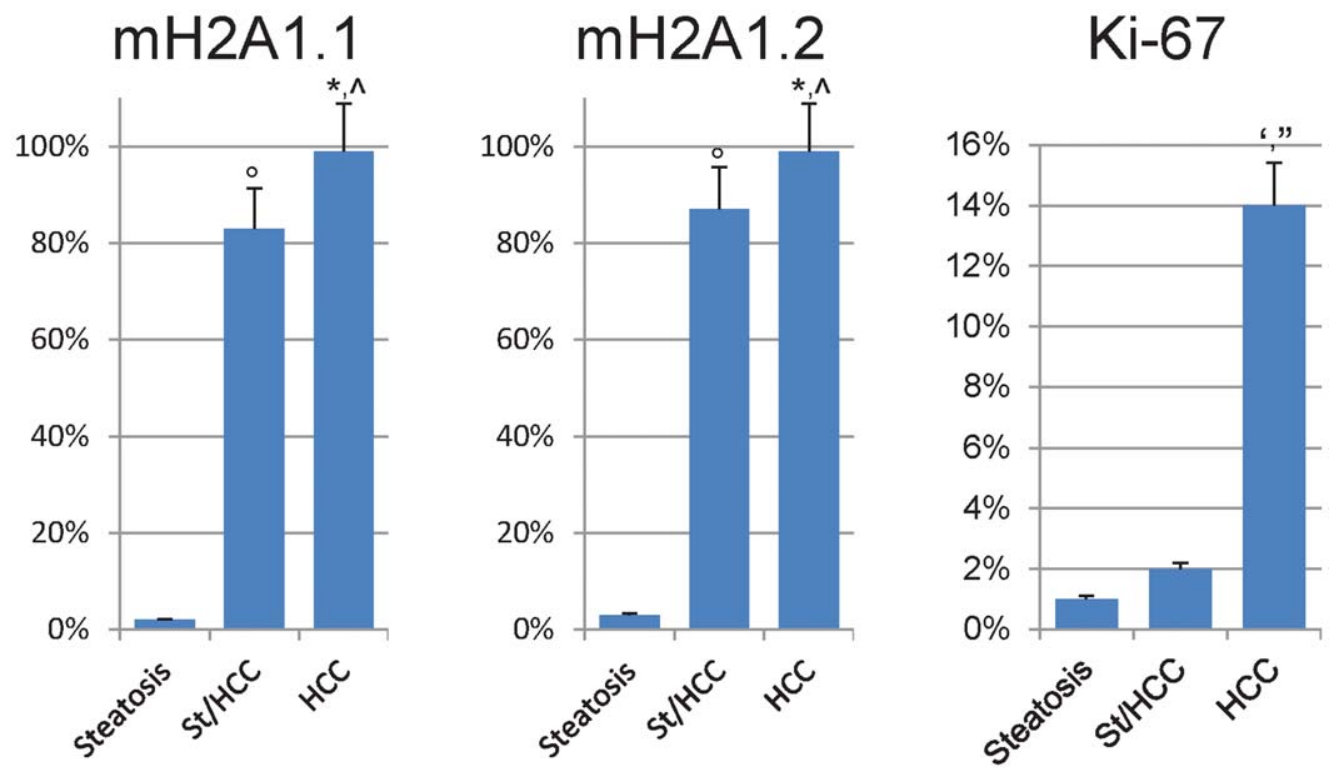

Figure 5. Histograms show statistical results for the evaluation of immunopositivity for macroH2A1.1, macroH2A1.2 and Ki-67 in samples of steatosis and HCC. In the latter both area with tumor and area of steatosis close to HCC (St/HCC) were examined. Significant differences $(p<0.005)$ in the percentage of positive nuclei were found for both MacroH2A1.1 and MacroH2A1.2 between St/HCC and steatosis $\left({ }^{\circ}\right)$, HCC and steatosis $\left(^{*}\right)$ and HCC and St/HCC () . Significant differences $(p<0.005)$ were also present for Ki- 67 between HCC and steatosis $\left({ }^{\prime}\right)$ and HCC and St/HCC (").

doi:10.1371/journal.pone.0054458.g005 
matory cytokines IL-6, TNF $\alpha$ and IL-1 $\beta$ mRNAs, while mice under ND did not (data not shown) [8].

To determine whether protein expression levels of macroH2Al.1 and macroH2A1.2 were altered in the context of steatosis or HCC, a histone extraction protocol was applied to the livers of mice fed a ND, fed with HF or injected with DEN and fed with $\mathrm{HF}$ (HF/DEN), followed by immunoblotting analysis. MacroH2A1.1 protein was found weakly expressed in the liver of $\mathrm{ND}$ or $\mathrm{HF}$ mice, while macroH2A1.2 expression was significantly increased in $\mathrm{HF}$-fed mice compared to ND-fed mice (Fig. 1B). Both macroH2A1.1 and macroH2A1.2 expression levels were highly enhanced in the HCG tissue of HF/DEN treated animals (Fig. 1B). Thus, in the HF/DEN dietary model of steatosis and $\mathrm{HCC}$, both macroH2Al isoforms were associated with cancer, whereas macroH2A1.2 is specifically increased in the presence of fat.

\section{MacroH2A1.1 and macroH2A1.2 Expression in the Liver of Liver-specific PTEN KO Mice}

Hepatocyte-specific PTEN deficiency results in steatosis and HCC in mice, at 10-16 and 74-78 weeks of age, respectively [27]. Expression of adipogenic and lipogenic genes, such as PPAR $\gamma$, is increased in the liver of hepatocyte-specific PTEN KO mice [27]. The transition from steatosis to HCG is accompanied in these animals by NASH and the appearance of liver adenomas at 40-44 weeks of age [27]. Paradoxically, PTEN being a negative regulator of insulin signalling [31], liver-specific PTEN KO mice displayed hepatic insulin hypersensitivity and increased systemic glucose tolerance [27]. We used this well established genetic model to study the expression of macroH2Al isoforms in hepatic steatosis and HCC. PTEN ${ }^{\text {flox/flox }}$ mice were crossed to AlbCre transgenic mice, in which expression of Cre is controlled by the promoter of the hepatocyte-specific gene albumin. Control PTEN ${ }^{\text {flox/flox }}$ mice and PTEN KO obtained by AlbCre-mediated deletion of both PTEN alleles were retained for experiments. Animals were sacrificed at 16 and 52 weeks of age for histological and biochemical analyses. As expected the liver of 16 weeks old PTEN KO mice showed extensive fat accumulation compared to 16 weeks old PTEN ${ }^{\text {flox/flox }}$ littermates (mean NAFLD score 4 versus 1, respectively), (Figure 2A). We assessed if the protein expression levels of macroH2A1.1 and macroH2A1.2 were altered in the context of steatosis or HCG in liver specific PTEN KO mice. Immunoblotting analysis on histone extract revealed that macroH2A1.1 levels were low both in the liver of PTEN ${ }^{\text {flox/flox }}$ mice and of 16 weeks old PTEN KO, while macroH2A1.2 expression was greatly enhanced in the liver of 16 weeks old PTEN $\mathrm{KO}$ compared to age-matched PTEN ${ }^{\text {flox/flox }}$ mice (Fig. 2B). Both macroH2Al.1 and macroH2Al.2 protein expression levels were massively increased in the HCG tissue of 52 weeks old PTEN KO mice (Fig. 2B). Similarly to the HF/DEN model, in the PTEN KO model of steatosis and HCC both macroH2Al isoforms associate with cancer, whereas macroH2A1.2 is specifically upregulated in the fatty liver.

\section{MacroH2A1.1 and macroH2A1.2 Expression in the Liver of Steatotic/HCC Patients}

Experiments were performed both in human samples with steatosis and samples with HCG (10 cases per each condition, Table 1). In the latter, areas with tumor and areas of steatosis close to HCG (St/HCG) were examined (Fig. 3A, Fig. 3B). Trichrome stain showed absence of fibrosis in all examined specimens. In particular, collagen was present only in portal space and, in limited amount, in perisinusoidal (Disse) spaces of lobule in both steatosis and St/HCC samples, as well as in the capsule that delimits HCC (Fig. 3B). Figure 4 shows representative immunostainings. Both macroH2Al.1 and macroH2A1.2 showed significant differences $(\mathrm{p}<0.005)$ in the percentage of positive nuclei between $\mathrm{St} / \mathrm{HCC}$ and steatosis, HCC and steatosis and HCC and St/HCC $(100 \%$ of tumor cells positive for either macroH2A1.1 or macroH2A1.2), when compared to steatosis $(<2 \%$ of hepatocytes positive for macroH2A1.1 and macroH2A1.2) (Fig 4, Fig. 5). The St/HCC areas were highly immunopositive for macroH2A1.1 and macroH2A1.2, displaying $83 \%$ versus $88 \%$ of positive nuclei, respectively $(p<0.05)$ (Fig 4, Fig. 5). Moreover, significant differences were also present for Ki-67 between HCC and steatosis and HCC and St/HCC (Fig. 4, Fig. 5). Finally, a number of nuclei in elements resembling to endothelial (sinusoidal) and perisinusoidal cells were also found positive both in steatosis and St/HCC areas (Fig. 4).

\section{Discussion}

In this study we report that the histone variant macroH2Al and its two splicing isoforms are strong markers of NAFLD-associated HCC, pointing to the importance of an epigenetic component in pathogenesis. One of the most striking epigenetic alterations that occur at the level of the chromatin is the exchange of the canonical $\mathrm{H} 2 \mathrm{~A}$ histone for histone variant macroH2A1, described nearly 20 years ago [32]. MacroH2Al can play either a positive or negative role in transcriptional regulation in a context-dependent manner, and it can control cell cycle and proliferation [33]. The two exon splicing variants of macroH2A1, macroH2Al.1 and macroH2A1.2, differ by just 3 aminoacids and differentially bind NAD metabolites [34]. As referred to earlier, KO mice for both macroH2A1 isoforms display insulin resistance, hepatic steatosis and an altered expression of hepatic genes involved in lipid metabolism (lipoprotein lipase, CD36 and others) [16,17], and alterations in the expression of macroH2A1.1 and macroH2A1.2 isoforms are associated to the occurrence/survival and/or the pathogenesis of various human cancers (lung, colon, melanoma) $[19,20,22]$. Our data show that both macroH2A1.1 and macroH2A1.2 protein expression levels are impressively increased in tumour tissue of human subjects presenting with HCC on a steatotic background without cirrhosis or fibrosis. The 10 patients studied with steatosis alone or with HCC show homogeneous and strongly consistent results. Immunohistochemistry analyses with specific antibodies showed that $100 \%$ of HCG nuclei were positive for macroH2Al isoforms compared to surrounding liver parenchyma or to the liver of steatotic subjects without HCG. This was observed also in the liver of HCG mice models, either dietary/ carcinogenic (HF/DEN) or genetic (PTEN KO), where HCC develops on the basis of a pre-existent NAFLD [8,27]. Independently of the causes underlying steatosis, an increase in macroH2Al.1 and macroH2Al.2 is strongly associated to HCC development in these experimental models. mRNA levels for macroH2Al.1 and macroH2A1.2 in the animal models and in liver biopsies from patients were variable and did not reflect the differences observed in the protein levels found in steatosis and HCC (data not shown). This is consistent with a previous study, indicating that, differently from most tissues analyzed to date, in the liver the mRNA splicing that generate the two isoforms of macroH2Al is not mirrored by changes in the protein levels [21]. We also found an interesting discrepancy between the NAFLD of the mouse models and that of the patients studied. In mice macroH2A1.2 expression is significantly increased in steatosis, whereas macroH2Al.1 is not; in human liver, mild content of fat alone was not associated to an increase of the isoforms $(<2 \%$ of 
positive nuclei for either isoform). However, in the steatotic areas of the liver proximal to the HCC tissue, high immunopositivity for both macroH2A1.1 and macroH2A1.2 was present, with a slightly greater number of positive hepatocytes for macroH2A1.2 (88\%) versus macroH2Al.1 (83\%). This would be consistent with an involvement of macroH2Al.1 and/or macroH2Al.2 in the pathogenic progression of steatotic liver to malignant HCG in man.

One limitation of the mouse studies is that the antibodies used for detection of the macroH2Al isoforms cannot be used in immunohistochemistry in this species. Consequently, immunoblotting data alone cannot distinguish between variations in macroH2A1.2 expression in the total liver histone extracts, if these are dependent from increases in hepatocyte expression or from other cell types, as non-hepatocyte cell types are stained in human liver immunohistochemistry (Fig. 4). Differences between HCC mouse models and HCG patients are multiple: mice do not develop HCC spontaneously, hence we manoeuvred to induce the disease either using an injection of DEN combined with a high fat diet, or by liver specific ablation of tumour suppressor PTEN, as previously described $[8,27]$. There are also conflicting issues about the role of genes involved in hepatocarcinogenesis (i.e., MET, NF$\kappa \mathrm{B}$, Stat3, Jnk, Shp2, and $\beta$-catenin) modelled in mice, which have arisen recently [35]. In any case, an increase of macroH2A1.2 observed only during fat accumulation may have metabolic implications. In this respect, the property of macroH2Al.1 in binding with very tight affinity NAD-derived metabolites, differ-

\section{References}

1. Calle EE, Rodriguez C, Walker-Thurmond K, Thun MJ (2003) Overweight, obesity, and mortality from cancer in a prospectively studied cohort of U.S. adults. N Engl J Med 348: 1625-1638.

2. Myers RP (2009) Noninvasive diagnosis of nonalcoholic fatty liver disease. Ann Hepatol 8 Suppl 1: S25-33.

3. Lou-Bonafonte JM, Arnal C, Osada J (2011) New genes involved in hepatic steatosis. Curr Opin Lipidol 22: 159-164.

4. Kantartzis K, Schick F, Haring HU, Stefan N (2010) Environmental and genetic determinants of fatty liver in humans. Dig Dis 28: 169-178.

5. Haybaeck J, Zeller N, Wolf MJ, Weber A, Wagner U, et al. (2009) A lymphotoxin-driven pathway to hepatocellular carcinoma. Cancer Cell 16: 295308.

6. He G, Karin M (2011) NF-kappaB and STAT3 - key players in liver inflammation and cancer. Cell Res 21: 159-168.

7. Mueller KM, Kornfeld JW, Friedbichler K, Blaas L, Egger G, et al. (2011) Impairment of hepatic growth hormone and glucocorticoid receptor signaling causes steatosis and hepatocellular carcinoma in mice. Hepatology 54: 13981409.

8. Park EJ, Lee JH, Yu GY, He G, Ali SR, et al. (2010) Dietary and genetic obesity promote liver inflammation and tumorigenesis by enhancing IL-6 and TNF expression. Cell 140: 197-208.

9. Das M, Garlick DS, Greiner DL, Davis RJ (2011) The role of JNK in the development of hepatocellular carcinoma. Genes Dev 25: 634-645.

10. Naugler WE, Sakurai T, Kim S, Maeda S, Kim K, et al. (2007) Gender disparity in liver cancer due to sex differences in MyD88-dependent IL-6 production. Science 317: 121-124.

11. Goldberg A, Allis CD, Bernstein E (2007) Epigenetics: a landscape takes shape. Cell 128: 635-638.

12. Doye NC, An W, Angelov D, Bondarenko V, Mietton F, et al. (2006) Mechanism of polymerase II transcription repression by the histone variant macroH2A. Mol Cell Biol 26: 1156-1164.

13. Ladurner AG (2003) Inactivating chromosomes: a macro domain that minimizes transcription. Mol Cell 12: 1-3.

14. Gamble M, Frizzell KM, Yang G, Krishnakumar R, Kraus WL (2010) The histone variant macroH2A1 marks repressed autosomal chromatin, but protects a subset of its target genes from silencing. Genes Dev 24: 21-32.

15. Creppe C, Janich P, Cantarino N, Noguera M, Valero V, et al. (2012) MacroH2Al regulates the balance between self-renewal and differentiation commitment in embryonic and adult stem cells. Mol Cell Biol 32: 1442-1452.

16. Boulard M, Storck S, Cong R, Pinto R, Delage,H, etal. (2010) Histone variant macroH2A1 deletion in mice causes female-specific steatosis. Epigenetics Chromatin 3: 8. [Epub ahead of print].

17. Changolkar L, Costanzi C, Leu NA, Chen D, McLaughlin KJ, et al. (2007) Developmental changes in histone macroH2A1-mediated gene regulation. Mol Cell Biol 27: 2758-2764. ently from macroH2A1.2 [34], is intriguing. NAD-derived metabolites such as $\mathrm{O}$-acetyl-ADP ribose (OAADPR) is generated by the enzymatic reaction catalyzed by SIRT1, a NAD-dependent protein deacetylase, whose activation is considered protective against cellular metabolic and oxidative stresses, and against aging $[36,37]$. Of note, liver-specific SIRT1 transgenic mice are protected against metabolic syndrome-associated HCC [38]. MacroH2A1.1 apparently suppresses growth of lung cancer cells and adenocarcinoma cells in a manner dependent on its ability to bind NAD-derived metabolites [21]. The presence of a metabolitebinding function in a chromatin component opens new potential connections between gene expression and lipid metabolism in the liver. Macro domains could also represent a novel tool for studying NAD metabolites and may be an attractive drug target [39].

\section{Acknowledgments}

We thank Dr. Wing Kin Syn for critical reading of the manuscript. We are grateful to Prof. Tak W. Mak (University Health Network, Toronto, Canada) for providing us with the Pten $^{\text {flox/flox }}$ mice.

\section{Author Contributions}

Conceived and designed the experiments: FC M. Foti GM M. Federici VP MV. Performed the experiments: FR AG CP LB MP AM NS. Analyzed the data: FC M. Foti GM M. Federici VP MV. Contributed reagents/ materials/analysis tools: FC M. Foti NS M. Federici. Wrote the paper: RW MV.

18. Pogribny IP, Tryndyak VP, Bagnyukova TV, Melnyk S, Montgomery B, et al. (2009) Hepatic epigenetic phenotype predetermines individual susceptibility to hepatic steatosis in mice fed a lipogenic methyl-deficient diet. J Hepatol 51: 176186.

19. Sporn JC, Kustatscher G, Hothorn T, Collado M, Serrano M, et al. (2009) Histone macroH2A isoforms predict the risk of lung cancer recurrence. Oncogene 28: 3423-3428.

20. Sporn JC, Jung B (2012) Differential Regulation and Predictive Potential of MacroH2A1 Isoforms in Colon Cancer. Am J Pathol 180: 2516-2526.

21. Novikov L, Park JW, Chen H, Klerman H, Jalloh AS, et al. (2011) QKIMediated Alternative Splicing of the Histone Variant MacroH2Al Regulates Cancer Cell Proliferation. Mol Cell Biol 31: 4244-4255.

22. Kapoor A, Goldberg MS, Cumberland LK, Ratnakumar K, Segura MF, et al. (2010) The histone variant macroH2A suppresses melanoma progression through regulation of CDK8. Nature 468: 1105-1109.

23. Vinciguerra M, Carrozzino F, Peyrou M, Carlone S, Montesano R, et al. (2009) Unsaturated fatty acids promote hepatoma proliferation and progression through downregulation of the tumor suppressor PTEN. J Hepatol 50: 1132 1141 .

24. Vinciguerra M, Sgroi A, Veyrat-Durebex C, Rubbia-Brandt L, Buhler LH, et al. (2009) Unsaturated fatty acids inhibit the expression of tumor suppressor phosphatase and tensin homolog (PTEN) via microRNA-21 up-regulation in hepatocytes. Hepatology 49: 1176-1184.

25. Vinciguerra M, Veyrat-Durebex C, Moukil MA, Rubbia-Brandt L, RohnerJeanrenaud F, et al. (2008) PTEN down-regulation by unsaturated fatty acids triggers hepatic steatosis via an NF-kappaBp65/mTOR-dependent mechanism. Gastroenterology 134: 268-280.

26. Vinciguerra M, Foti M (2008) PTEN at the crossroad of metabolic diseases and cancer in the liver. Ann Hepatol 7: 192-199.

27. Horie Y, Suzuki A, Kataoka E, Sasaki T, Hamada K, et al. (2004) Hepatocytespecific Pten deficiency results in steatohepatitis and hepatocellular carcinomas. J Clin Invest 113: 1774-1783.

28. Kleiner DE, Brunt EM, Van Natta M, Behling C, Contos MJ, et al. (2005) Design and validation of a histological scoring system for nonalcoholic fatty liver disease. Hepatology 41: 1313-1321.

29. Salamone F, Galvano F, Cappello F, Mangiameli A, Barbagallo I, et al. (2012) Silibinin modulates lipid homeostasis and inhibits nuclear factor kappa B activation in experimental nonalcoholic steatohepatitis. Transl Res 159: 477486.

30. Farina F, Cappello F, Todaro M, Bucchieri F, Peri G, et al. (2000) Involvement of caspase-3 and GD3 ganglioside in ceramide-induced apoptosis in Farber disease. J Histochem Cytochem 48: 57-62.

31. Vinciguerra M, Foti M (2006) PTEN and SHIP2 phosphoinositide phosphatases as negative regulators of insulin signalling. Arch Physiol Biochem 112: 89-104. 
32. Pehrson JR, Fried VA (1992) MacroH2A, a core histone containing a large nonhistone region. Science 257: 1398-1400.

33. Gamble MJ, Kraus WL (2010) Multiple facets of the unique histone variant macroH2A: From genomics to cell biology. Cell Cycle 9.

34. Kustatscher G, Hothorn M, Pugieux C, Scheffzek K, Ladurner AG (2005) Splicing regulates NAD metabolite binding to histone macroH2A. Nat Struct Mol Biol 12: 624-625.

35. Feng GS (2012) Conflicting roles of molecules in hepatocarcinogenesis: paradigm or paradox. Cancer Cell 21: 150-154.
36. Vinciguerra M, Fulco M, Ladurner A, Sartorelli V, Rosenthal N (2010) SirT1 in muscle physiology and disease: lessons from mouse models. Dis Model Mech 3: 298-303.

37. Vinciguerra M, Santini MP, Martinez C, Pazienza V, Claycomb WC, et al. (2012) $\mathrm{mIGF}-1 / \mathrm{JNK} 1 / \mathrm{SirT} 1$ signaling confers protection against oxidative stress in the heart. Aging Cell 11: 139-149.

38. Herranz D, Munoz-Martin M, Canamero M, Mulero F, Martinez-Pastor B, et al. (2010) Sirt1 improves healthy ageing and protects from metabolic syndromeassociated cancer. Nat Commun 1: 1-8.

39. Till S, Ladurner AG (2009) Sensing NAD metabolites through macro domains. Front Biosci 14: 3246-3258. 